\title{
Quantitative gaschromatographische Bestimmung von Gallensäuren als Trimethylsilyl-Äther-Ester-Derivate freier Gallensäuren $\left.{ }^{1}\right)$
}

\author{
Von P. BACK \\ Aus dem Pathologischen Institut der Universität München, Elektronenmikroskopische Abteilung \\ (Vorstand: Prof. Dr. F. Miller)
}

(Eingegangen am 18. März 1969)

Freie Gallensäuren lassen sich durch Reaktion mit N,O-Bis-trimethylsilylacetamid und Trimethylchlorsilan in einem einzigen Reaktionsschritt in Trimethylsilyl-Äther-Ester-Derivate überführen, welche auf Methylvinyl-Silikongummi als stationärer Phase aufgetxcnnt werden können. Das angegebene Verfahren zeichnet sich durch Raschheit und Reproduzierbarkeit aus und ist für die klinisch-chemische Analyse geeignet.

\section{The quantitative determination of free bile acids by the gas chromatography of their trimetbylsilyl-etber-ester derivatives}

Unconjugated bile acids react readily with bis-trimethylsilylacetamide and trimethylchlorosilane to give trimethylsilyl-ether-ester-derivatives. Gas-liquid-chromatographic separation of these compounds was partially achieved on a methyl-vinyl-silicone rubber as stationary phase. The method described seems to be suitable for analyses in the clinical laboratory.

Die Bestimmung freier Gallensäuren ist gaschromatographisch nach verschiedenen Methoden, welche sich im wesentlichen durch die Art der zur Analyse gelangenden Säurederivate voneinander unterscheiden, möglich. Während die Methylester infolge ihrer hohen Polarität zur Adsorption auf den verwendeten Trennsäulen neigen (1), eignen sich Doppelderivate der Säuren besser für die quantitative Bestimmung. Diese können nach Methylierung der Carboxylgruppe ent-. weder durch Veresterung der Hydroxylgruppen mit Hilfe von Trifluoracetanhydrid (2) oder Bildung der Trimethylsilyläther an diesen Positionen (3) hergestellt werden.

Die quantitative Gallensäure-Analyse mittels dieser Verfahren blieb jedoch bisher weitgehend auf chemische Laboratorien beschränkt. Der Grund hierfür liegt einmal in der relativ aufwendigen Methodik zur quantitativen Gewinnung der reinen Gallensäurenfraktionen $(4,5)$, zum anderen ist die Bildung der Doppelderivate nur bei strenger Beachtung der Reaktionsvorschriften und extremem Feuchtigkeitsausschluß aus Lösungsmitteln und Proben möglich. Die quantitative Bildung der Methylester gelingt mit Hilfe von Diazomethan sehr leicht (6), jedoch ist beispielsweise die vollständige 'Trimethylsilylierung nàch unserer Erfahrung nur möglich, wenn die Ester vorher mindestens für 24 Stunden im Ölpumpenivakuum scharf getrocknet werden.

Für die klinisch-chemische Analyse von Gallensäuren sollte daher ein Verfahren angestrebt werden, welches eine Vereinfachung der chemischen Reaktionsschritte bei der Bildung verdampfbarer Derivate und eine Verkürzung der Gesamtanalysenzeit mit sich bringt. Nach den Untersuchungen von HoRNING und Mitarbeitern $(7,8)$ ist mit dem Silylierungsreagens, N,O-Bis-(trimethylsilyl)acetamid (BTMSA), beschrieben von BrR-

1) Mit Unterstützung durch die Deutsche Forschungsgemeinschaft.
KOFER und Mitarbeitern (9), die Bildung von Trimethylsilylestern verschiedener biologisch interessanter Säuren möglich, welche sich für die Gaschromatographie eignen, jedoch liegen für Gallensäuren bisher keine näheren Angaben über die notwendigen Analysenbedingungen vor. Aufbauend auf diesen Befunden wird in dieser Arbeit ein vereinfachtes gaschromatographisches System für die quantitative Analyse einiger freier Gallensäuren beschrieben.

\section{Material und Methoden}

$3 \alpha-, 7 \alpha-, 12 \alpha-$ Trihydroxycholansäure (Cholsäure Merck AG,

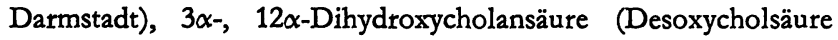

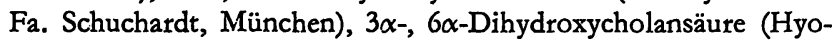
desoxycholsäure $\mathrm{Fa}$. Schuchardt, München) und $3 \alpha$-Hydroxycholansäure (Lithocholsäure $\mathrm{Fa}$. Schuchardt, München) wurden durch mehrfaches Umkristallisieren gereinigt. $3 \alpha-, 7 \alpha$-Dihydroxycholansäure (Chenodesoxycholsäure ,grade $A$ “, Calbiochem AG, Luzern) und $3 \alpha-, 6 \alpha-, 7 \alpha-$ Trihydroxycholansäure (Hyocholsäure), welche freundlicherweise durch Herrn Dr. H. Danielsson zur Verfügung gestellt wurde, wurden ohne vorherigen Reinigungsschritt verwendet.' $3 \alpha$-Hydroxy-12-keto-cholansäure wurde aus Desoxycholsäure synthetisiert (10). 3 $3 \alpha$-Hydroxy-7,12-diketo-

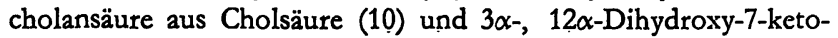
cholansäuremethylester aus Cholsäuremethylester (11). Die freie $3 \alpha-, 12 \alpha$-Dihydroxy-7-keto-cholansäure wurde nach alkalischer Hydrolyse und Atherextraktion nach Ansäuern erhalten. Die synthetisierten Keto-Galleṇäaruren waren noch nicht als gaschromatographisch rein anzusehen, sie enthielten kleinere Mengen anderer Keto-Gallensäuren, deren vorläufige Identifizierung an Hand der von MAKITA und Wells (3) angegebenen relativen Retentionszeiten für die Methylester-Trimethylsilyläther-Derivate erfolgte.

N,O-Bis-Trimethylsilylacetamid (BTMSA) war erhältlich über die Fa. Serva, Heidelberg. Trimethylchlorsilan (TMCS) wurde bezogen von Fa. Fluka, Buchs, Schweiz, ebendort auch Dimethyldichlorsilan (DMDCS).

Für die gaschromatographische Analyse wurde der Gaschromatograph 402 der Fa. Hewlett-Packard, ausgerüstet mit einem Flammenionisationsdetektor, verwendet. Dic Registrierung erfolgte mit dem Schreiber 7128 A (Hewlett-Packard). Dic Injektionen wurden dosiert mit einer $10 \mu /$ Hamilton-Nadel (Hamilton Bo- 
naduz, Schweiz). Folgende Trennsäulen wurden getestet: $4 \%$ SE 30 auf silyliertem Chromosorb WAW 80-100 mesh (Fa. Schuchardt, München), $225 \times 0,3 \mathrm{~cm}, 0,75 \%$ CHDMS (Cyclohexanodimethanolpolysuccinat $\mathrm{Fa}$. Serva, Heidelberg) auf silyliertem Chromosorb WAW, $330 \times 0,3 \mathrm{~cm}$, und $3,8 \%$ UCC-W 982 auf Diatoport S, 80-100 mesh, $118 \times 0,3 \mathrm{~cm}$ (HewlettPackard, Böblingen). Die U-förmig gebogenen, mit Dimethyldichlorsilan vorbehandelten Glassäulen waren mit Silikongummiringen am Injektor- und Detektoranschlu $ß$ abgedichtet. Trägergas war Helium mit einer Flußgeschwindigkeit von $60 \mathrm{~m} / / \mathrm{Min}$. bei einem Eingangsdruck von 3,5 atü. Nachgeteinigter Wasserstoff diente als Brenngas. Die Arbeitstemperaturen lagen für die verwendeten Säulen zwischen $235^{\circ}$ und $250^{\circ}$, die Injektortemperatur wurde auf $280^{\circ}$ und die Detektortemperatur auf $300^{\circ}$ eingestellt.

Linearität des Detektorsignals war bei Testung mit $5 \alpha$-Cholestan (Aldrich Chemical Co. Inc. Milwaukee Wis.) im Bereich von $10-2000 \mathrm{ng}$ injizierter Substanz gegeben.

\section{Vorbereitung der Trennsäulen}

U-förmige, gewendelte Glassäulen mit einem inneren Durchmesser von $0,3 \mathrm{~cm}$ wurden in verschiedener Länge durch die $\mathrm{Fa}$. Neumann, München hergestellt. Die Säulen wurden mit dest. Toluol, dest. Methanol und dest. Aceton vorgereinigt und nach Trocknung mit wenigen $\mathrm{ml}$ dest. Dimethyldichlorsilan durchspült. Nach erneuter Trocknung im Luftstrom wurden die Säulen mit Trägermaterial gefüllt.

\section{Herstellung der Säulenfiillung}

Etwa $20 \mathrm{~g}$ Chromosorb WAW 80-100 mesh wurden in dest. Wasser aufgeschwemmt. Kleinere Partikel wurden nach dem Absetzen mit dem Uberstand dekantiert. Dieser Waschprozeß wurde zweimal wiederholt. Anschließend wurde das feuchte Trägermaterial auf einer Absaugvorrichtung trockengesaugt und mehrmals mit dest. Aceton gewaschen. Der gewaschene Träget wurde auf einer Glasplatte vorsichtig ausgebreitet und über Nácht bei $100^{\circ}$ im Trockenschrank getrocknet. Der noch warme Träger wurde am nächsten $T a g$ in einen $\dot{2}-\dot{l}$-Rundkolben eingebracht, welcher über einen Dreiweghahn mit einem 100-ml-Rundkolben und einer Wasserstrahlpumpe verbunden war. In den $100-\mathrm{ml}$ Rundkolben wurden $8 \mathrm{~m} l$ Dimethyldichlorsilan gegeben, dann wurde der große Kolben evakuiert. Nach erfolgter Evakuierung wurden die Hähne so geöffnet, $\mathrm{da} B$ die beiden Rundkolben in Verbindung gelangten und über dem Trägermaterial eine Atmosphäre von DMDCS entstand. Wenn der Siedevorgang im kleinen Rundkolben zum Stillstand gekommen war, wurden die Hähne wieder geschlossen und der große Kolben wurde erneut evakuiert, um den entstandenen Chlorwasserstoff zu entfernen. Dieser Vorgang wurde unter leichtem. Schwenken der Trägerpartikel etwa zehnmal wiederholt. Daran schloß sich eine mehrmalige Waschung mit Methanol bis zur Neutralität an. Das gewaschene Trägermaterial wurde wiederum bei $100^{\circ}$ getrocknet. Die so behandelten Trägerteilchen müssen sich im Schwimmversuch auf der Wasseroberfläche spreiten und dürfen nicht absinken.

Die flüssige Phase wurde nach dem von Horning und Mitarbeitern beschriebenen Verfahren (12) auf den Träger gebracht. Die erhaltene Beladung wurde in Gewichtsprozenten nach wiederholter Waschung eines Aliquots des Trägers mit dem für die flüssige Phase verwendeten Lösungsmittel bestimmt.

\section{Bildung der Trimetbylsilyl-Äther-Ester-Derivate}

Bis zu $2 \mathrm{mg}$ der Gallensäuren wurden einzeln oder in einer Mischung in trockene Reagenzgläser eingebracht. Nach Durch-
Abb. 1

Trennung von Gallensäuren als TrimethylsilylÄther-Ester auf einer 3,8 proz. UCC-W 982 Trennsäule $(118 \times 0,3 \mathrm{~cm})$. Arbeitstemperatur $239^{\circ}$. Trägergasfluß $60 \mathrm{~m} l / \mathrm{Min}$. Helium

$1=5 \alpha$-Cholestan, $2=$ Cholesterin-Trimethylsilyläther, $\quad 3=$ Lithocholsäure, $4=$ Desoxycholsäure, $\quad 5=$ Cholsäure, $\quad 6=$ Hyodesoxycholsäure, $\quad 7=3 x$-Hydroxy-12-ketocholansäure, $8=3 \alpha-, 12 \alpha$-Dihydroxy-7-ketocholansäure, $9=$ $3 x$-Hydroxy-7,12-diketocholansäure
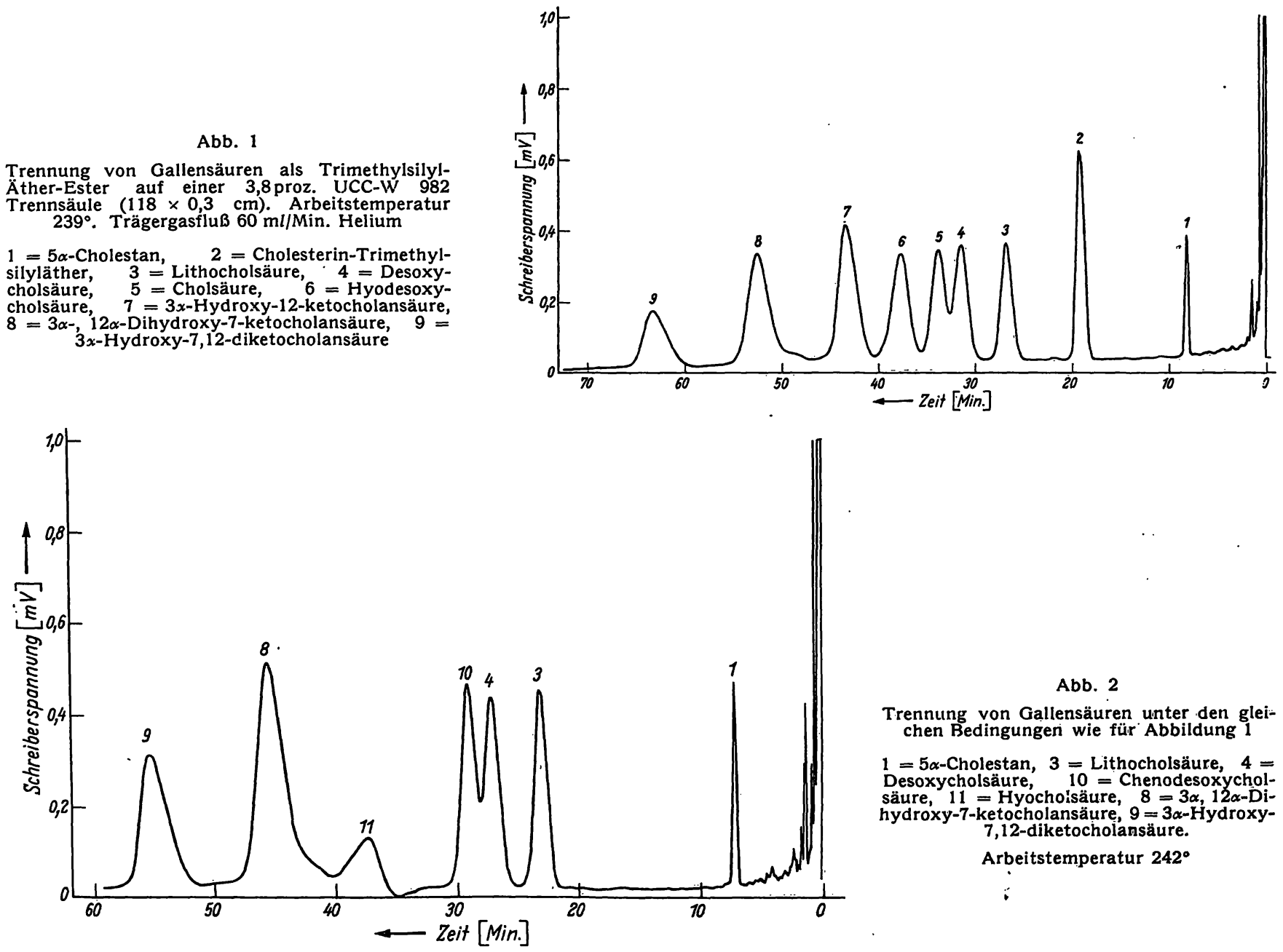

Abb. 2

Trennung von Gallensäuren unter den gleichen Bedingungen wie für Abbildung

$1=5 \alpha$-Cholestan, $3=$ Lithocholsäure, $4=$ Desoxycholsäure, $10=$ Chenodesoxycholsäure, $11=$ Hyochoisäure, $8=3 \alpha, 12 \alpha$-Dihydroxy-7-ketocholansäure, $9=3 \alpha$-Hydroxy7,12-diketocholansäure. Arbeitstemperatur $242^{\circ}$ 
Abb. 3

Gas-Chromatogramm der sauren ätherlöslichen Fraktion eines Patientenserums nach alkalischer Hydrolyse der konjugierten Gallensâuren und Extraktion der Hauptmenge der neutralen Steroide. Analysenbedingungen wie für Abbildung 1 . Arbeitstemperatur $240^{\circ}$.

2 = Cholesterin-Trimethylsilyläther

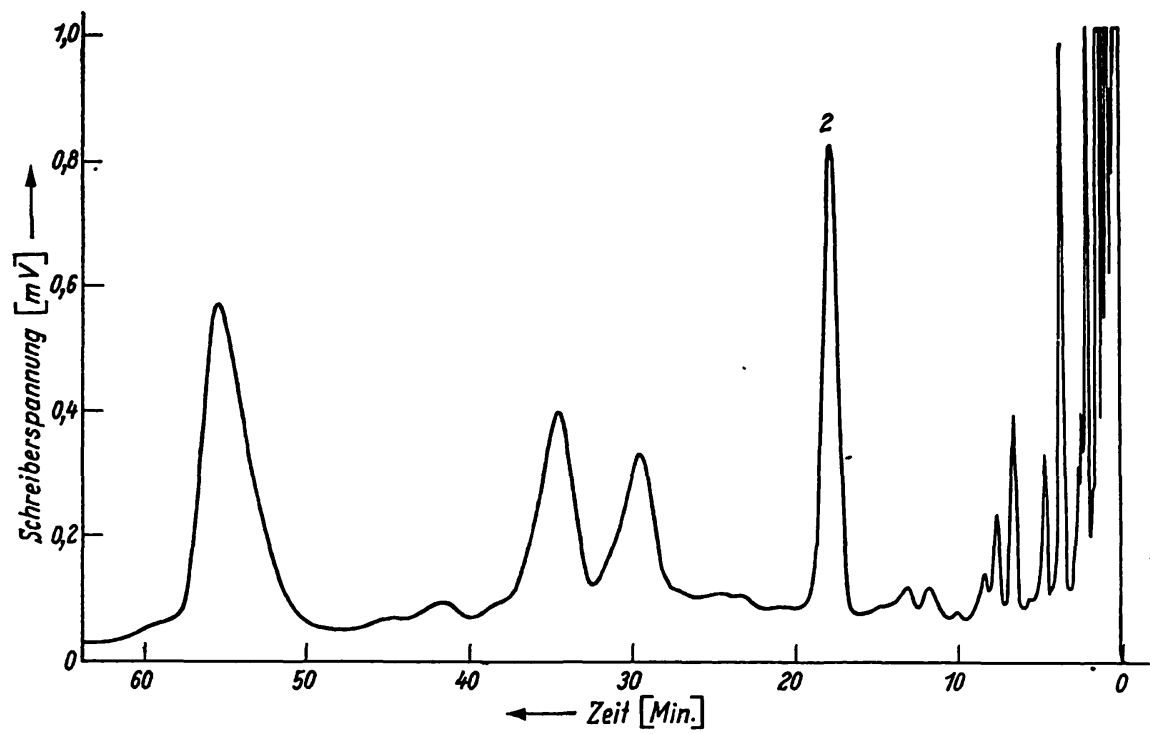

spülen des Reagenzglases mit einem über Calciumchlorid getrockneten Stickstoffgasstrom wurden rasch $200 \mu l$ des Silylierungsgemisches zugesetzt, welches aus 4 Teilen BTMSA und 1 Teil TMCS bestand. Das Reagenzglas wurde sofort hermetisch verschlossen und bei Raumtemperatur stehengelassen. Die ersten Injektionen in den Gaschromatographen erfolgten nach $15 \mathrm{Min}$. Reaktionszeit. Das Auftreten von einem einzigen Gipfel der jeweiligen Säure zeigte die Vollständigkeit der Reaktion an. Stehenlassen der Proben im verschlossenen Gefäß über drei Tage führte zu keinen Zersetzungserscheinungen. Wiederholtes Offnen der Reagenzgläser jedoch bewirkte rasch eine hydrolytische Spaltung der Esterbindung, wie von anderen Autoren ebenfalls beobachtet werden konnte (9).

\section{Ergebnisse}

Die Chromatogramme in Abbildung 1 und 2 zeigen Trennleistungen, welche mit einer 3,8proz. UCC-W 982 Trennsäule von $118 \mathrm{~cm}$ Länge erzielt werden. Hervorzuheben ist die deutliche Trennung von Cholesterin und Gallensäuren, welche nach dem von Makita und Wells angegebenen Verfahren nicht möglich war. Dort zeigte Cholesterin annähernd die gleiche Retentionszeit wie Cholsäure. Die quantitative Extraktion von Cholesterin aus biologischen Proben, welche auf Gallensäuregehalt untersucht werden sollen, erweist sich daher als nicht mehr so kritisch für die Analyse. Es bedeutet einen großen Vorteil, wenn die Zahl der Extraktionsvorgannge gering gehalten werden kann, da gerade durch diese auch bei Verwendung von inneren Standards für den Extraktionsgrad Unsicherheiten in die Analyse eingeführt werden. Dieser Vorteil ist aus Abbildung 3 ersichtlich, in welcher das Chromatogramm der sauren ätherlöslichen Fraktion eines $\mathrm{Pa}-$ tientenserums- wiedergegeben ist. Die Aufbereitung des Serums erforderte lediglich die folgenden Schritte: Nach alkalischer Hydrolyse des Serums werden die neutralen Steroide mit Äther extrahiert, der Ätherextrakt wird mit kleinen Volumina dest. Wassers gewaschen, die vereinigten wäßrigen Phasen werden auf den $\mathrm{pH}$-Wert 1 gebracht und wiederum mit Äther extrahiert. Der Ätherextrakt wird über wasserfreiem Natriumsulfat kurz getrocknet und im Vakuum eingedampft. Nach Zugabe des Silylierungsgemisches wird die Analyse durchgeführt.

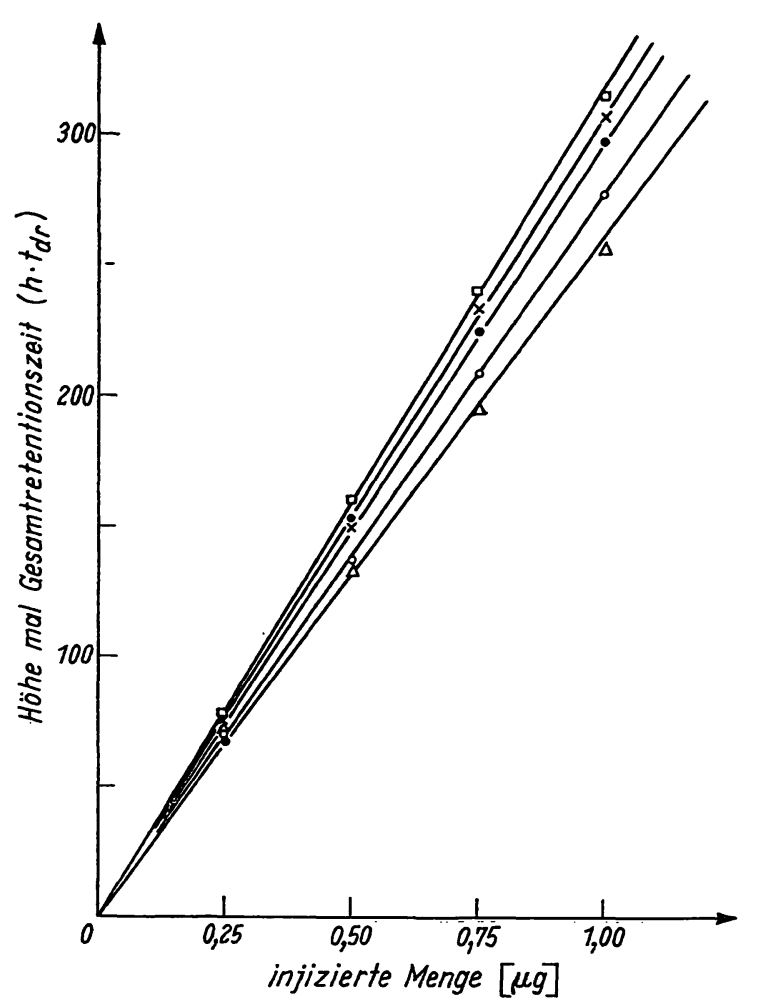

Abb. 4 $\left.z e i t, h \cdot t_{d r}\right)$ von der Menge injizierter Substanz. Mengen bezogen auf eingewogene freie Säuren

ㅁ 3x, 6x-Dihydroxycholansäure

$\times 3 x, 7 \alpha$-Dihydroxycholansäure

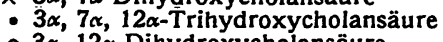

$\triangle$ 3x-Hydroxycholansâure

Die relativen Retentionszeiten der TrimethylsilylÄther-Ester-Derivate einiger Gallensäuren auf 3,8proz. UCC-W 982 sind in Tabelle 1 zusammengestellt. Bezugssubstanz ist das Lithocholsäurederivat.

$\mathrm{Da}$ die Einstellung der Temperaturbedingungen und Trägergasflußgeschwindigkeiten leichten Schwankungen unterliegen kann, insbesondere, wenn der Gaschromatograph aus wirtschaftlichen Gründen nicht auf Dauerbetrieb gehalten werden kann und eine Neueinstellung der Betriebsbedingungen von einem Tag auf den anderen erforderlich ist, empfiehlt sich für die quantitative Auswertung einzelner Gipfel in isothermen Gaschromato-
Abhängigkeit des Schreibersignals (Gipfelhöhe mal Gesamtretentions-

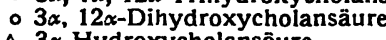


Tab. 1

Relative Retentionszeiten einzelner Gallensäuren als TrimethylsilylÄther-Ester Derivate bezogen auf Lithocholsäure-Trimethylsilyläther Trimethylsilylester $(1,00)$. Trennbedingungen s. Abbildung 1

\begin{tabular}{|c|}
\hline $\begin{array}{l}\text { 3a-Hydroxycholansäure } \\
3 \alpha-, 12 a-D i h y \text { droxycholansäure } \\
3 \alpha-, 7 a-D i h y d r o x y c h o l a n s a ̈ u r e \\
3 a-, 6 x-D i h y d r o x y c h o l a n s a ̈ u r e \\
3 x-, 7 a-, 12 \alpha-\text { Trihydroxycholansäure } \\
3 x-, 6 x-, 7 a-T r i h y d r o x y c h o l a n s a ̈ u r e \\
3 a-\text { Hydroxy-12-ketocholansäure } \\
\text { 3x,12a-Dihydroxy-7-ketocholansäure } \\
\text { 3a-Hydroxy-7,12-diketocholansäure }\end{array}$ \\
\hline
\end{tabular}

grammen das Ausmessen durch die Näherungsmethode Höhe (h) mal Gesamtretentionszeit $\left(t_{d r}\right)$ (13). Diese Schnellmethode liefert sehr gut $\mathrm{zu}$ reproduzierende Werte bei Verwendung eines massenmessenden Detektors wie des Flammenionisationsdetektors, wenn die Trennsäule nicht überlastet wird. Bei der Anfertigung von Eichkurven, wie sie für einige Gallensäuren in Abbildung 4 dargestellt sind, ist es erforderlich, durch Beimischung eines inneren Standards Korrekturfaktoren für Volumenschwankungen, welche bei der Abmessung des Injektionsvolumens in der Hamilton-Nadel auftreten können, einzuführen. $5 \alpha$-Cholestan hat sich hierbei als brauchbare Standardsubstanz erwiesen.

Die erhaltenen stoffspezifischen Geraden werden als Eichgeraden für die quantitativen Analysen in Proben mit verschiedener Zusammensetzung bekannter Einzelkomponenten verwendet. Die Identifizierung unbekannter Einzelkomponenten jedoch ist in der Regel erst dann möglich, wenn sich an die gaschromatographische Auftrennung eine massenspektrometrische Analyse anschließt (14).

\section{Diskussion}

Mit der Einführung von Bis-(trimethylsilyl)acetamid (BTMSA) als Silylierungsagens kann die quantitative Analyse von Gallensäuren stark vereinfacht werden. In einem Reaktionsschritt können alle Hydroxylgruppen veräthert und die Carboxylgruppen verestert werden, wodurch nicht nur eine wesentliche Zeitersparnis erzielt wird, sondern auch die Reproduzierbarkeit bei Analysen dieser Substanzen aus biologischem Material erhöht wird. Insbesondere erweist sich der Wegfall der Methylierung, welche den Umgang mit dem giftigen und leicht explosiblen Diazomethan erforderte, als Vorteil, weil die Trockenheit der Methylester von kritischer Bedeutung für die erfolgreiche quantitative Bildung der Trimethylsilylätherbindung durch das Gemisch Hexamethyldisilazan/Trimethylchlorsilan/Pyridin war. Es kommt bei der Reaktion mit BTMSA auch nicht zu dem Präzipitat von Ammoniumchlorid, welches das Analysenergebnis durch Inhomogenität der zu untersuchenden Probe verfälschen konnte. Für die Gaschromatographie der Trimethylsilyl-Äther-Ester-Derivate erweist sich die Phase UCC-W 982, ein MethylVinyl-Silikongummi, besser geeignet als die Phasen SE 30 oder CHDMS, auf welcher Verluste der Komponenten beobachtet werden konnte. SE 30 zeigte keine effektive Auftrennung der Einzelkomponenten. Für die Anwendung im klinischen Laboratorium erscheint die relativ kurze Zeit, die für eine Analyse benötigt wird, als Hauptvorteil. Nach alkalischer Hydrolyse des zu untersuchenden Materials werden lediglich zwei Extraktionsschritte benötigt, um zur gallensäurehaltigen Fraktion zu gelangen, welche nach Eindampfen und kurzer Reaktionszeit die für die Gaschromatographie erforderlichen flüchtigen Derivate ergibt. Fettsäuren, welche ebenfalls extrahiert werden, zeigen unter den angewandten Untersuchungsbedingungen so kurze Retentionszeiten, $\mathrm{da}$ sie nicht störend wirken. $\mathrm{Da}$ die beiden Hauptgallensäuren Cholsäure und Chenodesoxycholsäure nur geringe Unterschiede in ihrer relativen Retentionszeit besitzen (s. Tab. 1), ist ihre Trennung in dem vorliegenden System noch nicht möglich, jedoch ist diese bei Verwendung längerer Säulen durch Erhöhung der Trennstufenzahl zu erwarten. Der Nachteil längerer Analysenzeiten kann dabei durch Erhöhung der Arbeitstemperatur teilweise wettgemacht werden, da UCC-W 982 nach unserer Erfahrung auch noch bei Temperaturen bis zu $280^{\circ}$ verwendet werden kann. Allerdings wird die Gesamtlebenszeit einer"solchen Säule bei diesen Temperaturen herabgesetzt. Bei der klinischen Analyse von Funktionsstörungen, bei welchen es zum erhöhten Auftreten von Gallensäuren im Serum kommen kann, wird das beschriebene gaschromatographische System möglicherweise von Bedeutung sein. Untersuchungen hierzu sind in Vorbereitung.

\section{Literatur}

1. Bloomfield, D. K., Analytic. Chem. 34, 737 (1962). - 2. Kuksis A. und B. A. Gordon, Canad. J. Biochem. 41, 1355 (1963). - 3. Makita, M. und W. W. Wells, Analytic. Biochem. 5, 523 (1963). - 4. SJövall, J., Meth. Biochem. Analysis 12, 97 (1964). - 5. Grundy, S. M., E. H. Ahrens jr. und T. A. MretTINEN, J. Lipid Res. 6, 397 (1965). - 6. Schlenk, H. und J. L. Gellerman, Analytic. Chem. 32, 1412 (1960). - 7. Horning, E. C., M. G. Horning, N. Ikekawa, E. M. Chambaz, P. I. JaAkonmaki und C. J. W. Brooks, J. Gas Chromatogr. 5, 283 (1967). - 8. Horning, M. G., E. A. Boucher und A. M. Moss, J. Gas Chro- matogr. 5, 297 (1967). - 9. Brrkofer, L., A. RitTer und W. Gressler, Angew. Chem. 75, 93 (1963). - 10. Kaziro, K. und T. Shrmada, Hoppe-Seyler's Z. physiol. Chem. 249, 220 (1937). 11. Matkovics, B. und B. Samuelsson, Acta chem. scand. 16, 683 (1962). - 12. Horning, E. C., E. A. Moscatelli und C. C. SweElex, Chem. \& Ind. (London) 751 (1959). - 13. KaIser, R., Chromatographie in der Gasphase Bd. 4 Bibliographisches Institut Mannheim (1965). - 14. ENeroth, P., K. Hellström und R. Ryhage, J. Lipid Res. 5, 245 (1964).
Dr. med. Peter Back 78 Freiburg im Breisgau Hugstetterstr. 55 


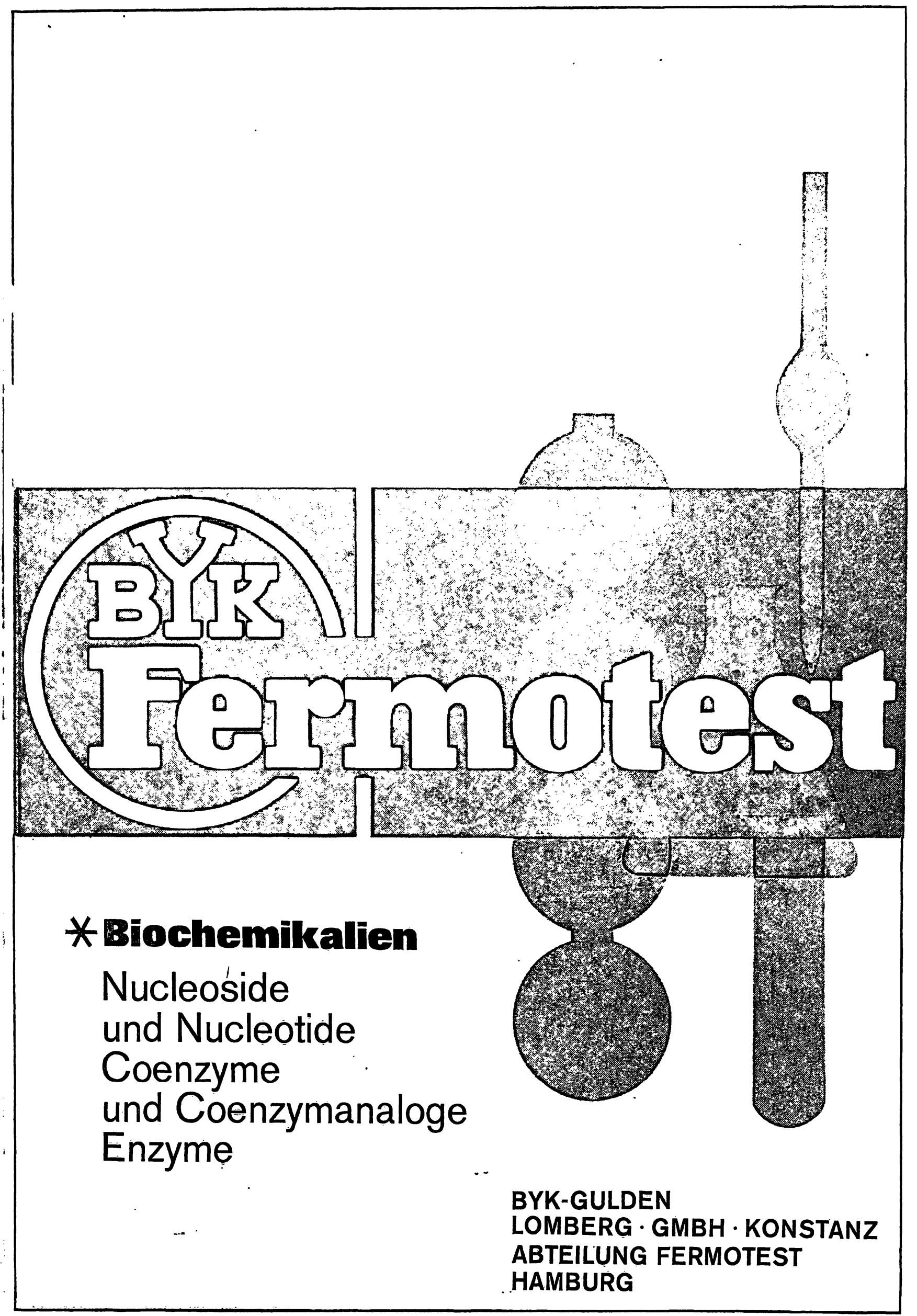




\title{
(UGerin) AABORGLAS
}

\author{
E-M M
}

\section{EINMAL serologische Ausblas-Messpipetten}

Kein Säubern,

Spülen, Trocknen, keine fehlerhaften Ergebnisse durch unsaubere, unsterile, beschädigte Pipetten.

Für die Arbeit in medizinischen, pharmazeutischen und biochemischen Laboratorien sowie für die Strahlenund Radiochemie bedeuten diese E-MIL-Einmalpipetten einen echten Fortschritt.

Unterlagen erhalten Sie beim Fachhändler oder schreiben Sie an:

\section{QUICKFIT LABORGLAS GMBH}

Wiesbaden-Schierstein - Hüttenstraße 8 Telefon (061 21) 64091 . FS 04186503 